\title{
Discrete Elastica
}

\author{
Alfred M. Bruckstein, ${ }^{\star}$ Robert J. Holt, and Arun N. Netravali \\ Bell Laboratories, Murray Hill, New Jersey 07974, USA
}

\begin{abstract}
This paper develops a discrete approach to the design of planar curves that minimize cost functions dependent upon their shape. The curves designed by using this approach are piecewise linear with equal length segments and obey various types of endpoint constraints.
\end{abstract}

\section{Introduction}

Planar curves that minimize energy functionals of the form $\int\left(\alpha \kappa^{2}+\beta\right) d s$, where $\kappa$ is the curvature and $s$ is arc length, subject to various types of boundary conditions are called elastica, following Euler's 1744 work titled "De Curvis Elastica" [1]. Such curves, also called nonlinear splines in the industrial design context, are important in a variety of applications and have been thoroughly studied in the past by Max Born [2] and A. Love [3]. Recently, due to renewed interest in such curves in computer graphics, CAD, and as shape completion curves in image analysis and computer vision, several papers have appeared, dealing with their effective computation and applications; see e. g. [4-12]. Even mathematicians seem to have recently rediscovered these interesting objects [13-17]. Since the actual computation of nonlinear splines turns out to be quite difficult, in CAD people turned to simpler polynomial splines or rational curves (NURBS) to address problems of shape design, see e.g. [18, 19], and some interesting ideas involving interpolation and design with optimized bi-arc and real algebraic curves which have also appeared [20,21].

In this paper we shall address the problem of designing discretized, piecewise linear curves minimizing functionals of various types that could be regarded as discretized versions of the "curve energy" considered in the context of splines. We shall argue that solving the nonlinear equations that result from considering "discrete elastica" is a feasible alternative to curve design. Furthermore we claim. that almost nothing is lost in the process of discretization since it is seldom the case that the differential equations arising from the the continuous optimization problem can be explicitly solved. This being the case, those equations must be solved by numerical integration, hence we shall end up with a discretized solution anyhow. We therefore propose to start with a discretized problem and concentrate on numerical procedures that can effectively solve them.

Let us first set the stage for the optimization problems considered. We shall always deal with planar polygonal curves (or poly-lines) with equal-length links

* On sabbatical from Dept. of Computer Science, Technion - IIT 32000, Haifa, ISRAEL. 
(or segments) specified via a set of points $\left\{\mathbf{P}_{i}\right\}_{i=0,1, \ldots, N, N+1}$ so that $d\left(\mathbf{P}_{i+1}, \mathbf{P}_{i}\right)$ $=l$ for all $i \in\{0, \ldots, N\}$ (see Figure 1).

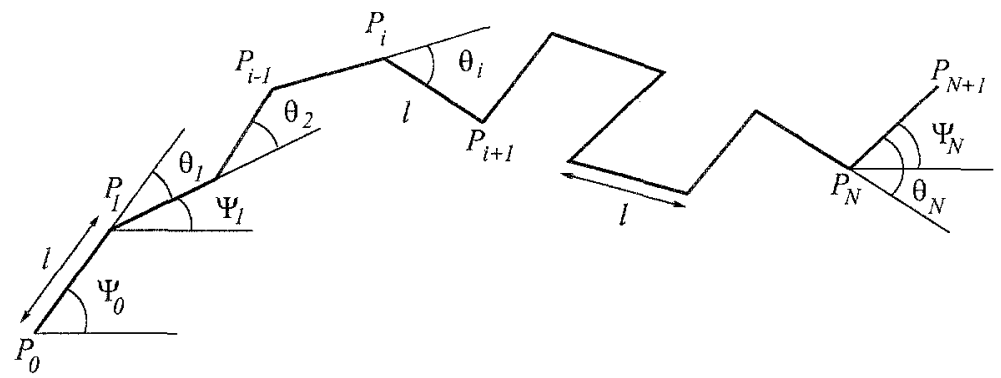

Fig. 1. A polygonal curve of equal-length segments.

The turn angle at $\mathbf{P}_{i}$, defined as $\Psi_{i}-\Psi_{i-1}$, where $\Psi_{i}$ is the angle of $\overline{\mathbf{P}_{i} \mathbf{P}_{i+1}}$ with the $x$-axis (as in Figure 1 ), will be denoted by $\theta_{i}$, and the curves we shall design will attempt to make these curves as smooth as possible by minimizing an "energy functional" that increases with the increase of these turn angles $\left|\theta_{i}\right|$. In fact the "discrete curvature" at $\mathbf{P}_{i}$ of a polyline as considered above could be defined as $\kappa_{i}=\theta_{i} / l$ and hence a reasonable "energy functional" candidate would be

$$
\sum_{i=1}^{N}\left[\alpha\left(\frac{\theta_{i}}{l}\right)^{2}+\beta\right] l \triangleq E_{1}(\theta, l)
$$

where $\theta=\left\{\theta_{1}, \ldots, \theta_{N}\right\}$, mimicking $\int\left(\alpha \kappa^{2}+\beta\right) d s$ from the continuous case.

Sometimes one may wish to design curves that are not as smooth as possible but as short as possible for some given boundary conditions, and perhaps some additional restrictions on their curvature; see e. g. Dubins [22]. For such cases we can replace the energy functionals to be optimized with other types of cost functions. Indeed, if, for example, we want to limit the "discrete curvature" of the polylines considered to some $|K|$ we can define a penalty function that increases sharply if the discrete curvature $|\theta / l|$ approaches $|K|$, (for example) as follows:

$$
C_{K, m}\left(\frac{\theta}{l}\right)=\left(\frac{\theta}{l K}\right)^{2 m} .
$$

Here, if $m$ is large, $C_{K, m}(\theta / l)$ becomes huge when $|\theta / l|$ exceeds $|K|$. With such a penalty function we could design curves to optimize

$$
E_{K, m}(\theta, l) \triangleq \sum_{i=1}^{N}\left[\alpha C_{K, m}\left(\theta_{i} / l\right)+\beta\right] l
$$

for given boundary conditions.

Boundary conditions that will be of interest to us here are the origin and endpoint of the polygonal curves and the directions of the first and last links. We shall assume that the first link starts at $(0,0)$ in the plane and ends at 
some $(L, 0)$, clearly without any loss of generality, since we would like to have at least Euclidean invariance built into the design process! Moreover, since we shall sometimes aim to have similarity (i. e. scale) invariance, we may also allow $l$ and/or $L$ to vary freely, according to the case.

Next we shall examine several cases of discrete elastica designs. Those will include solving the following type of optimization problem:

minimize $E(\boldsymbol{\theta}, l)$ subject to:

predetermined $\Psi_{0}$ and $\Psi_{N} \quad \sum_{i=0}^{N} l \cos \Psi_{i}=L \quad \sum_{i=0}^{N} l \sin \Psi_{i}=0$,

where $\Psi_{j} \triangleq \Psi_{0}+\sum_{i=1}^{j} \theta_{i}$ for $j=1, \ldots, N$. There are several parameters in such optimization problems that can be either set a priori or optimized. The number of links $N+1$ and the length of each link (jointly determining the length of the curve as $(N+1) l)$ are such parameters. $L$ is also free if we are interested in similarity invariant elastica, since it scales directly with $l$.

\section{2 "Classical" Discrete Elastica}

We shall first discuss polygonal elastica (poly-elastica) that minimize

$$
E(\theta, l)=\sum_{i=1}^{N}\left[\alpha\left(\frac{\Psi_{i}-\Psi_{i-1}}{l}\right)^{2}+\beta\right] l
$$

subject to

$\Psi_{0}$ and $\Psi_{N}$ predetermined $\quad \sum_{i=0}^{N} l \cos \Psi_{i}=L \quad \sum_{i=0}^{N} l \sin \Psi_{i}=0$.

Using the Lagrange multiplier technique we consider the cost function

$$
\begin{aligned}
& \mathcal{G}\left(\boldsymbol{\Psi}, \lambda_{1}, \lambda_{2}, l ; L, N, \alpha, \beta\right) \\
& \quad=\alpha l \sum_{i=1}^{N}\left(\frac{\Psi_{i}-\Psi_{i-1}}{l}\right)^{2}+N \beta l+\lambda_{1}\left(l \sum_{i=0}^{N} \cos \Psi_{i}-L\right)+\lambda_{2} l \sum_{i=0}^{N} \sin \Psi_{i}
\end{aligned}
$$

where $\Psi$ denotes the vector of unknown angles $\left\{\Psi_{1}, \ldots, \Psi_{N-1}\right\}$. Taking derivatives with respect to the $N+2$ variables $\Psi_{1}, \ldots, \Psi_{N-1}, \lambda_{1}, \lambda_{2}$, and $l$, leads to the system of equations

$$
\left\{\begin{array}{c}
\frac{2 \alpha\left(-\Psi_{j-1}+2 \Psi_{j}-\Psi_{j+1}\right)}{l^{2}}-\lambda_{1} \sin \Psi_{j}+\lambda_{2} \cos \Psi_{j}=0, j=1, \ldots, N-1, \\
l \sum_{i=0}^{N} \cos \Psi_{i}-L=0, \quad \sum_{i=0}^{N} \sin \Psi_{i}=0, \\
-\alpha \sum_{i=1}^{N}\left(\frac{\Psi_{i}-\Psi_{i-1}}{l}\right)^{2}+N \beta+\lambda_{1} \sum_{i=0}^{N} \cos \Psi_{i}+\lambda_{2} \sum_{i=0}^{N} \sin \Psi_{i}=0 .
\end{array}\right.
$$


The above case addresses the problem of design of polylines that allows the boundary conditions to set/choose an optimal link-length 6 . We could, however, address a different type of optimization too: we could set $l=1$ and let the endpoint $(L, 0)$ be free to settle anywhere on the positive $x$-axis. Indeed we can readily imagine a "discrete" physical spline made from inelastic sections, or links, of length 1 , connected with elastic joints where the bending potential energy depends on the turn angle $\theta$ as $\alpha \theta^{2}$. The problem we would address in this case would be to determine the lowest energy configuration of such a "spline" when the first link is forced to make an angle of $\Psi_{0}$ with the $x$-axis and the last link an angle of $\Psi_{N}$ with its endpoint free to slide along the positive $x$-axis (see Figure 2).

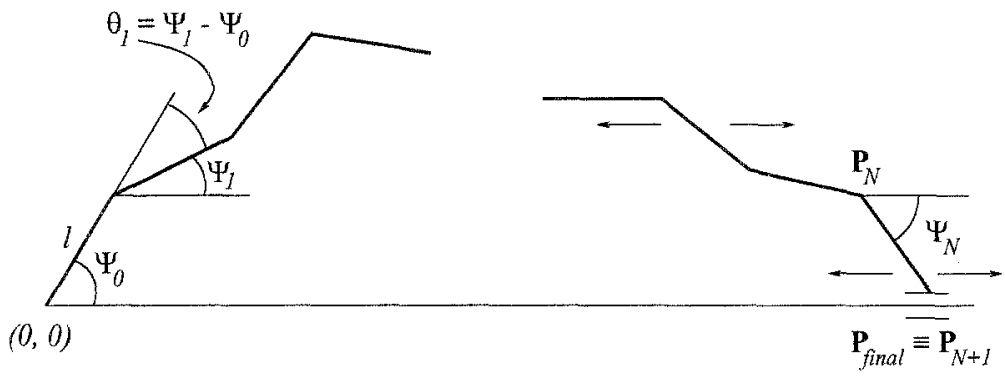

Fig. 2. The terminal point may "slide" along the $x$-axis.

With $l=1$ we have the following optimization problem:

$$
\begin{aligned}
& \text { minimize } E(\theta, l)=\sum_{i=1}^{N}\left(\Psi_{i}-\Psi_{i-1}\right)^{2} \\
& \text { subject to } \sum_{i=0}^{N} \sin \Psi_{i}=0 \quad \sum_{i=0}^{N} \cos \Psi_{i}>0 .
\end{aligned}
$$

Again, Lagrange multipliers were used to form the expression

$$
\mathcal{F}(\boldsymbol{\Psi}, \lambda ; N)=\sum_{i=1}^{N}\left(\Psi_{i}-\Psi_{i-1}\right)^{2}+\lambda \sum_{i=0}^{N} \sin \Psi_{i} .
$$

Taking derivatives with respect to the $N$ variables $\Psi_{1}, \ldots, \Psi_{N-1}$, and $\lambda$ leads to the system of equations

$$
\left\{\begin{array}{c}
2\left(-\Psi_{j-1}+2 \Psi_{j}-\Psi_{j+1}\right)+\lambda \cos \Psi_{j}=0, j=1, \ldots, N-1, \\
\sum_{i=0}^{N} \sin \Psi_{i}=0 .
\end{array}\right.
$$

This optimization problem was considered in [10], and solved via a shooting method. It represents a discrete version of a scale-invariant version of minimal energy curves. Indeed, it was proved in [10] that $\int d s \cdot \int \kappa^{2} d s$ is a scale-invariant 
energy measure that leads to circular interpolations for symmetric endpoint conditions, a very desirable property indeed.

The main motivation for introducing the modified energy functional in [10] was the consideration of a counterintuitive result of Horn asserting that the least energy curve (minimizing $\int \kappa^{2} d s$ ) which starts vertically up at $(0,0)$ and arrives vertically down at $(1,0)$ is not a semicircle [8]. This result is due to the use of a cost function that does not penalize the curve length and is not scale invariant. Later, Moreton and Séquin also realized the advantage of similarity invariant cost functionals based on curvature and curvature variation in the design of curves, see [11]. The cost functions proposed by them were $\int \kappa^{2} d s$ and $\int(d \kappa / d s)^{2} d s$ for Euclidean invariant elastica and $\left(\int d s \cdot \int \kappa^{2} d s\right)$, as in [10], and $\left(\int d s\right)^{3}\left(\int(d \kappa / d s)^{2} d s\right)$ for scale invariant versions. We shall briefly return to some minimal variation curve design ideas in a later section.

\section{Discrete Elastica with Hard Limits on Turn}

In this section we address the problem of optimizing interpolation curves for other types of cost functions. In particular we shall be interested in penalizing curvatures in such a way that the resulting polylines will have turns, or "discrete curvatures," limited to an a priori given range. To obtain this result we can solve for polygonal elastica that minimize, say

$$
E_{K, m}(\boldsymbol{\theta}, l)=\sum_{i=1}^{N}\left[\alpha\left(\frac{\Psi_{i}-\Psi_{i-1}}{l K}\right)^{2 m}+\beta\right] l
$$

subject to the same conditions as before.

The Lagrange approach leads here to a nonlinear system of equations that displays the necessary condition for optimality, i. e. for

$$
\begin{aligned}
& \mathcal{G}\left(\boldsymbol{\Psi}, \lambda_{1}, \lambda_{2}, l ; K, L, m, N, \alpha, \beta\right) \\
& =\sum_{i=1}^{N}\left[\alpha\left(\frac{\Psi_{i}-\Psi_{i-1}}{l K}\right)^{2 m}+\beta\right] l+\lambda_{1}\left[\sum_{i=0}^{N} l \cos \Psi_{i}-L\right]+\lambda_{2}\left[\sum_{i=0}^{N} l \sin \Psi_{i}\right]
\end{aligned}
$$

taking derivatives with respect to the $N+2$ variables $\Psi_{1}, \ldots, \Psi_{N-1}, \lambda_{1}, \lambda_{2}$, and $l$, leads to the following system of nonlinear equations generalizing (2):

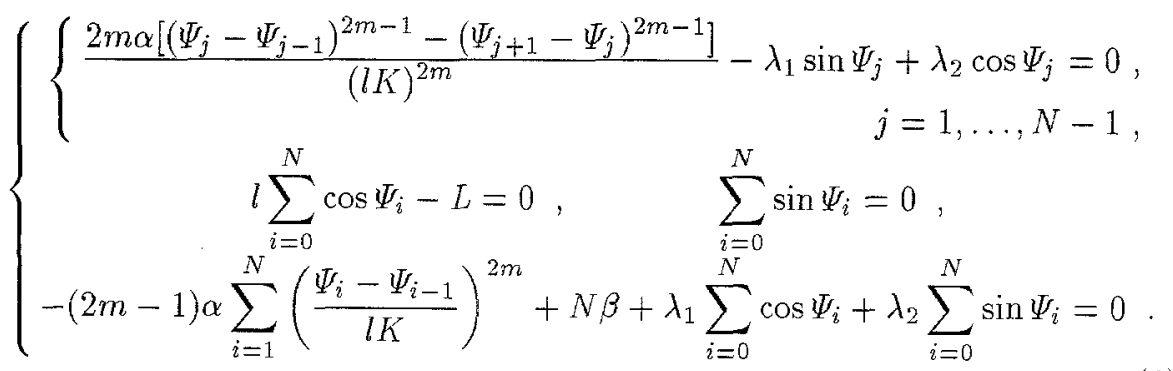


Note that if $\left(\boldsymbol{\Psi}^{*}, \lambda_{1}^{*}, \lambda_{2}^{*}, l^{*}\right)$ is a solution for the set of parameters $(K, L, m, N$, $\alpha, \beta)$, then $\left(\boldsymbol{\Psi}^{*}, \lambda_{1}^{*}, \lambda_{2}^{*}, c l^{*}\right)$ is a solution for the set of parameters $(K / c, c L, m, N$, $\alpha, \beta)$. Thus the solution curve scales with $L$ as long as $L K$ is held constant.

As previously, we could set $l$ to 1 and free the condition $l \sum_{i=0}^{N} \cos \Psi_{i}=L$ to $\sum_{i=0}^{N} \cos \Psi_{i}>0$. However, this condition alone, in conjunction with a penalty function that allows all turns less than $K$ for free (almost - for high $m$ 's) could lead to some peculiar returns of the curve to the $x$-axis. Indeed, minimizing (5) with $l=1$ and $\beta=0$ subject to $\sum_{i=0}^{N} \sin \Psi_{i}=0$ leads to

$$
\Psi_{j+1}=\Psi_{j}+\left[\left(\Psi_{j}-\Psi_{j-1}\right)^{2 m-1}+\lambda_{2} \frac{K^{2 m}}{2 m} \cos \Psi_{j}\right]^{\frac{1}{2 m-1}} .
$$

Note however that as $m \rightarrow \infty$ the terms $\left[\left(\Psi_{j}-\Psi_{j-1}\right) / K\right]^{2 m}$ will all tend to zero if $\left|\Psi_{j}-\Psi_{j-1}\right|<K$, hence all sequences of $\Psi_{j}$ obeying $\left|\Psi_{j}-\Psi_{j-1}\right|<K$ and $\sum_{i=0}^{N} \sin \Psi_{i}=0$ will be equally good. Therefore to make the problem well defined we need to impose an additional constraint. With similarity invariance considerations in view, we require the minimization of

$$
\sum_{i=1}^{N} \alpha\left(\frac{\Psi_{i}-\Psi_{i-1}}{K}\right)^{2 m}+\frac{\beta}{\sum_{i=0}^{N} \cos \Psi_{i}}
$$

subject to

$$
\sum_{i=0}^{N} \sin \Psi_{i}=0, \quad \quad \sum_{i=0}^{N} \cos \Psi_{i}>0 .
$$

(find interpolant with $l=1$ and maximum $L$ so that one has the greatest $L /[(N+$ 1)]] ratio!)

Lagrange multipliers were used to form the expression

$$
\mathcal{F}(\boldsymbol{\Psi}, \lambda ; K, m, N, \alpha, \beta)=\alpha \sum_{i=1}^{N}\left(\frac{\Psi_{i}-\Psi_{i-1}}{K}\right)^{2 m}+\frac{\beta}{\sum_{i=0}^{N} \cos \Psi_{i}}+\lambda \sum_{i=0}^{N} \sin \Psi_{i}
$$

Taking derivatives with respect to the $N$ variables $\Psi_{1}, \ldots, \Psi_{N-1}$, and $\lambda$ leads to this system which generalizes (4):

$$
\begin{gathered}
\frac{2 m \alpha\left[\left(\Psi_{j}-\Psi_{j-1}\right)^{2 m-1}-\left(\Psi_{j+1}-\Psi_{j}\right)^{2 m-1}\right]}{K^{2 m}}+\frac{\beta \sin \Psi_{j}}{\left(\sum_{i=0}^{N} \cos \Psi_{i}\right)^{2}}+\lambda \cos \Psi_{j}=0, \\
j=1, \ldots, N-1, \\
\sum_{i=0}^{N} \sin \Psi_{i}=0 .
\end{gathered}
$$


This optimization problem imposes the requirement to have $\sum_{i=0}^{N} \cos \Psi_{i}$, i. e. the excursion in the positive $x$-direction, as large as possible for a curve with given length $(N+1) l$. This means that if we scale the curve so as to have $\Delta x=1$, we shall have the polygonal curve of minimal length that obeys the (similarity invariant) local turn condition $\left(\left|\Psi_{j+1}-\Psi_{j}\right|<K\right)$. Or, in other words, the curve with the best (total length) $/(\Delta x$-excursion) ratios among all interpolating curves with limited turn.

The first problem with which we dealt in this section of the paper has a continuous counterpart: an old result of Dubins (see [22]) states that minimal length curves with a constraint on the (average) curvature and prescribed initial and terminal positions and tangents are always composed of a circular arc (with radius determined by the maximum curvature allowed), a linear segment, and another circular arc (or a subset of these three pieces). As seen in the next section, the numerical method we developed indeed yielded (for high $m$ 's) such interpolants. In fact, this happened for both types of optimization problems considered above.

\section{Numerical Experiments with Discrete Elastica}

In this section we discuss in detail the various problems and the numerical solutions corresponding to the four cost functions discussed in Sections 2 and 3. We also show several families of curves illustrating how changing some of the parameters affects the minimal cost curves for the various cost functions.

\subsection{Euclidean-invariant Elastica}

The discrete Euclidean-invariant elastica curves are solutions of the optimization problem (1). The solutions are found by solving the system (2), and this was accomplished by using the technique discussed for the turn/curvature limited elastica in Section 5.3 and substituting in $K=1$ and $m=1$. The program was written with the symbolic manipulation program Maple [23], and uses the Newton-Raphson method directly on the system (2).

Figure 3 shows the effect on varying the terminal angle while holding the other parameters constant at $\Psi_{0}=135^{\circ}, L=10, N=32, \alpha=1, \beta=1$.

Figure 4 shows the effect of $\alpha$ and $\beta$ on the results. As the ratio $\beta / \alpha$ increases, the contribution of the length of the curve to the cost function increases, and as a result the length of the curve decreases. This phenomenon is readily seen in Figure 4, which shows curves for $\beta / \alpha=0.1,1.0$, and 10. The other parameters are fixed at $\Psi_{0}=180^{\circ}, \Psi_{N}=180^{\circ}, L=10$, and $N=32$.

Figure 5 shows the effect on varying $N$, one less than the number of links, while holding the other parameters constant at $\Psi_{0}=45^{\circ}, \Psi_{N}=-135^{\circ}, L=6$, $\alpha=1, \beta=1$. There is very little change in the curve for values of $N$ larger than 64. 


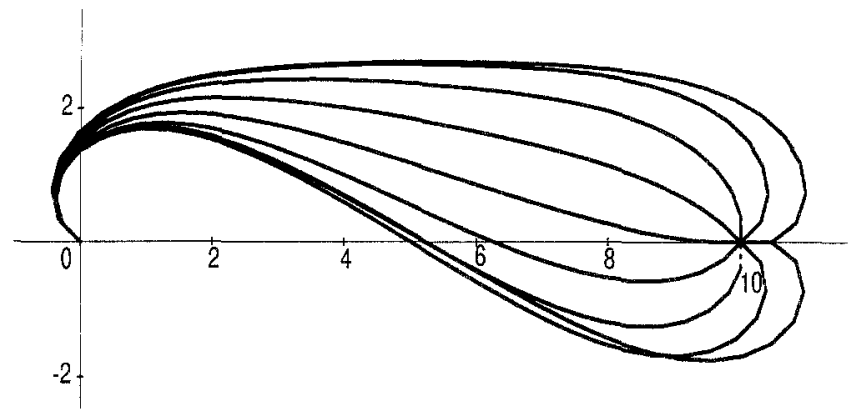

Fig. 3. Discrete Euclidean elastica with $\Psi_{0}=135^{\circ}, \Psi_{N}=-180^{\circ},-135^{\circ}, \ldots, 180^{\circ}$.

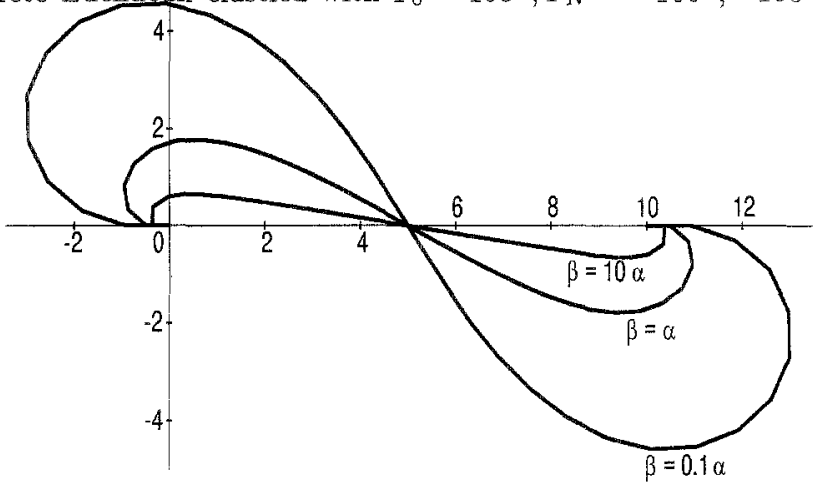

Fig. 4. Discrete Euclidean elastica with $\Psi_{0}=180^{\circ}, \Psi_{N}=180^{\circ}$, and $\beta / \alpha=0.1,1,10$.

\subsection{Similarity-invariant Elastica}

As in [10] we are interested here in solutions of the minimization problem (3).

Another program using Maple was written to solve this problem, specifically the system (4), for a wide range of parameter values. These were solved by using the technique for the "similarity-invariant" curvature limited elastica discussed in detail in Section 5.4 and substituting in $K=1, m=1, \alpha=1$, and $\beta=0$.

Figure 6 shows the effect on varying the terminal angle while holding the other parameters constant at $\Psi_{0}=135^{\circ}, N=32$.

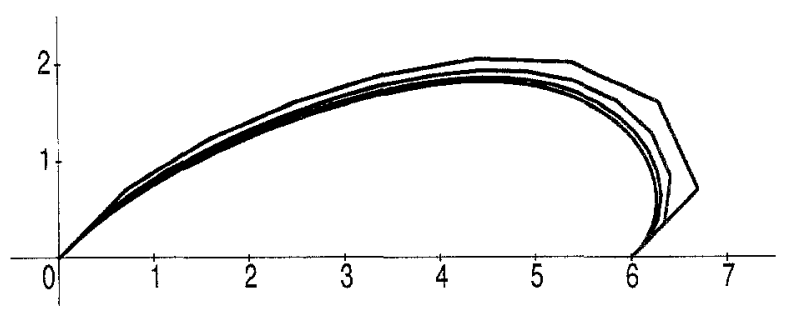

Fig. 5. Discrete Euclidean elastica with varying $N$. From top to bottom at $x=5$, these are the curves for $N=8,16,32$, and 64 respectively. 


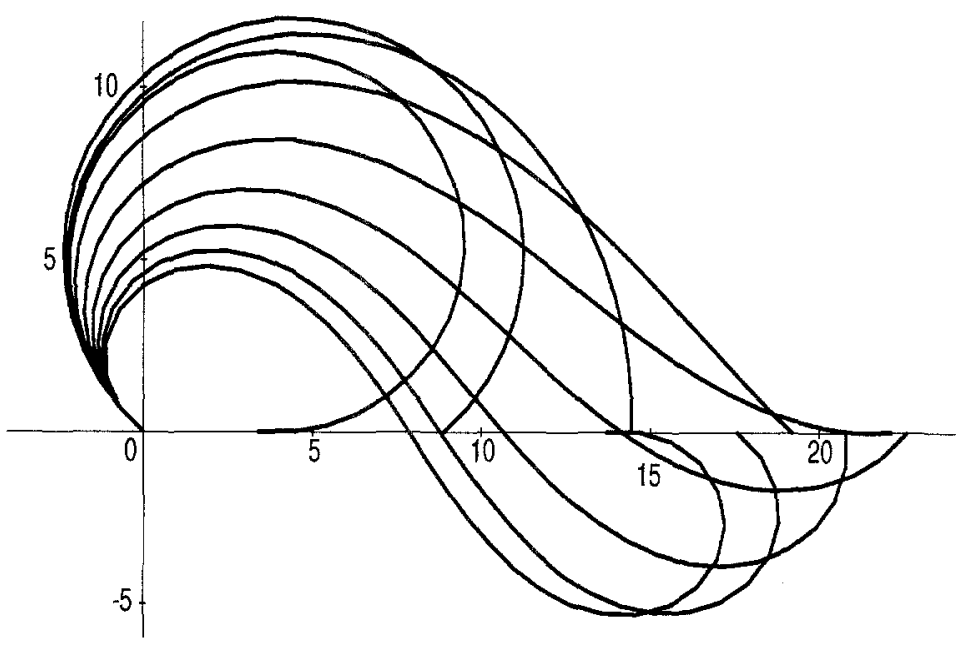

Fig. 6. Discrete similarity-invariant elastica with $N=32, \Psi_{0}=135^{\circ}, \Psi_{N}=-180^{\circ}$, $-135^{\circ}, \ldots, 180^{\circ}$.

Figure 7 shows the same results as in Figure 6, but with all of the distances scaled so that $L=x_{N+1}=1$. This has the effect of changing the length of each link from 1 to $1 / x_{N+1}=1 / \sum_{i=0}^{N} \cos \Psi_{i}$.

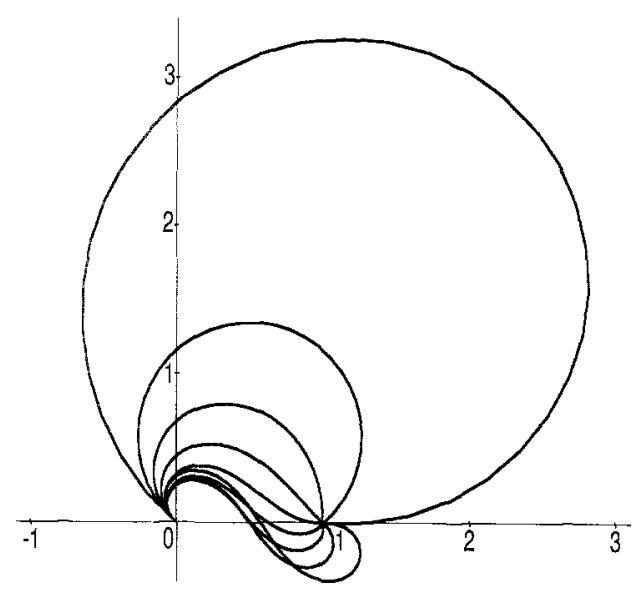

Fig. 7. Normalized similarity-invariant elastica with $N=32, \Psi_{0}=135^{\circ}, \Psi_{N}=-180^{\circ}$, $-135^{\circ}, \ldots, 180^{\circ}$.

Figure 8 shows the effect on varying $N$, one less than the number of links, while holding the other parameters constant at $\Psi_{0}=45^{\circ}$ and $\Psi_{N}=-135^{\circ}$.

Figure 9 shows the same results as in Figure 8 , but with all of the distances scaled so that $x_{N+1}=1$. The curves converge to a smooth path as $N$ increases. The limiting curve is not of circle-line-circle type since $m=1$ here; the circleline-circle behavior appears only for large $m$. 


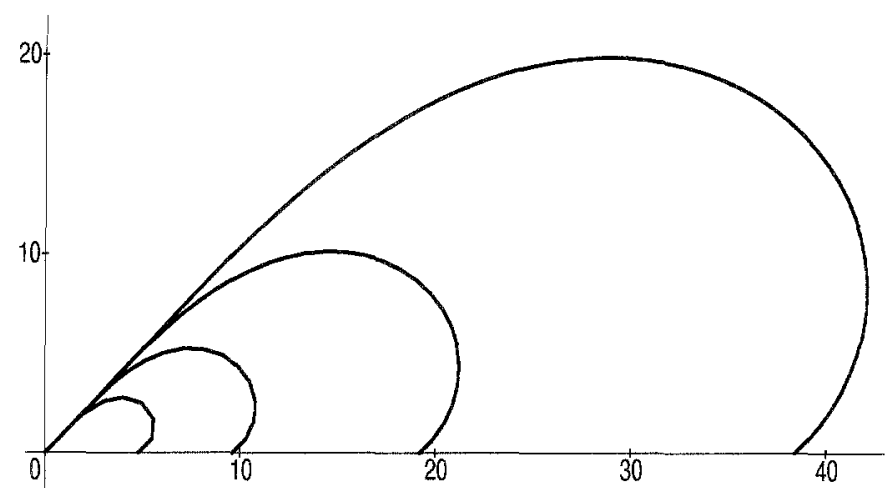

Fig. 8. Similarity-invariant elastica, with varying $N$. In order of increasing length, these are the curves for $N=8,16,32$, and 64 respectively.

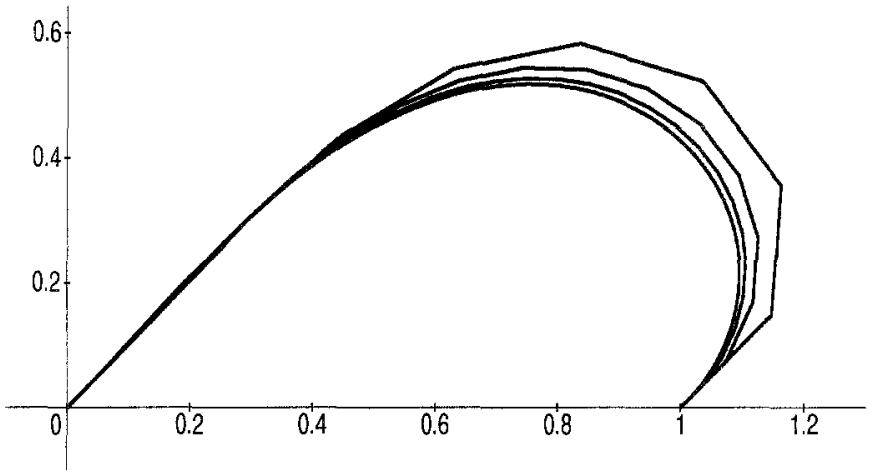

Fig. 9. Normalized similarity-invariant elastica with varying $N$. From top to bottom at $x=0.8$, these are the curves for $N=8,16,32$, and 64 respectively.

\subsection{Curvature Hard-limited Elastica}

As we saw in Section 4, here we want to solve the minimization problem (5). We wrote a program using the symbolic manipulation program Maple to solve the system (6) for many parameter values. The system of equations was solved using the Newton-Raphson method with an initial guess close to the solution for the continuous case, as suggested by [22]. That is, an approximation to a circle-line-circle type curve that meets the required boundary conditions is first found. We went to this trouble obtaining a good initial guess so that the NewtonRaphson method will converge to the correct answer, and in not a large number of iterations. In most of the examples fewer than ten iterations were required.

Figure 10 shows the effect on varying the terminal angle while holding the other parameters constant at $\Psi_{0}=135^{\circ}, L=21, N=32, K=0.4, m=100$, $\alpha=1, \beta=1$.

Figure 11 shows the effect of $\alpha$ and $\beta$ on the results when $m=10$. As the ratio $\beta / \alpha$ increases, the length of the curve decreases, but to much less a degree 


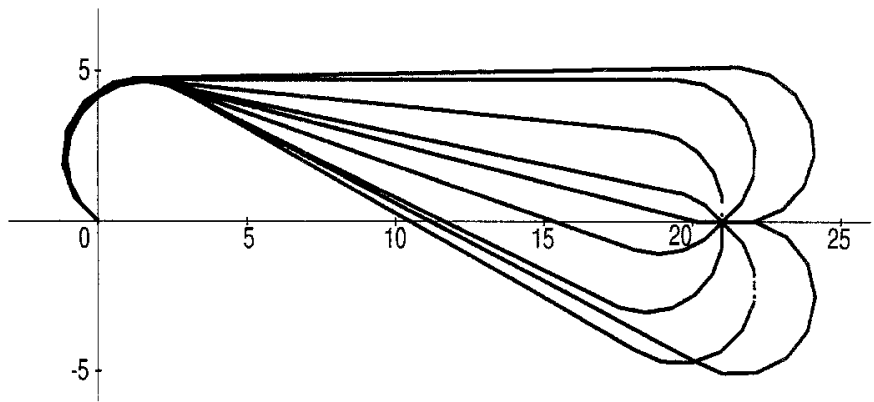

Fig. 10. Minimal length curvature-limited curves with $m=100, \Psi_{0}=135^{\circ}$, $\Psi_{N}=-180^{\circ},-135^{\circ}, \ldots, 180^{\circ}$.

than in Figure 4 when $m$ was 1 . This phenomenon may be seen in Figure 11 , which shows curves for $\beta / \alpha=0.1,1.0$, and 10 . When $m$ gets larger the curves for different ratios of $\beta / \alpha$ resemble each other more closely. The other parameters are fixed at $\Psi_{0}=90^{\circ}, \Psi_{N}=-135^{\circ}, K=6, L=1$, and $N=24$.

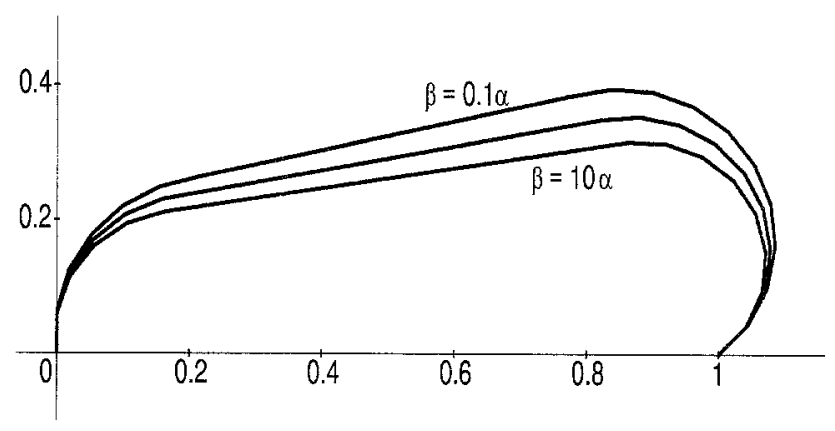

Fig. 11. Curvature-limited elastica with $\Psi_{0}=90^{\circ}, \Psi_{N}=-135^{\circ}$, and $\beta / \alpha=0.1,1,10$. The middle curve is the one obtained when $\beta=\alpha$.

\section{4 "Similarity invariant" Turn-limited Elastica}

Here we want to solve the minimization problem (7). We wrote another Maple program to solve the corresponding system (8) for a wide range of parameter values. These were solved by a technique very similar to that in described in detail in the previous section. The major difficulty arising in this case is that we do not know the value of $L$, or $x_{N+1}$, beforehand, so we cannot make the same computations as before. However, we found that we obtained acceptable results when we made an initial guess based on the approximation $L \approx N$. 
Figure 12 shows the effect on varying the terminal angle while holding the other parameters constant at $\Psi_{0}=135^{\circ}, K=0.4, m=100, N=32, \alpha=1$, $\beta=1$.

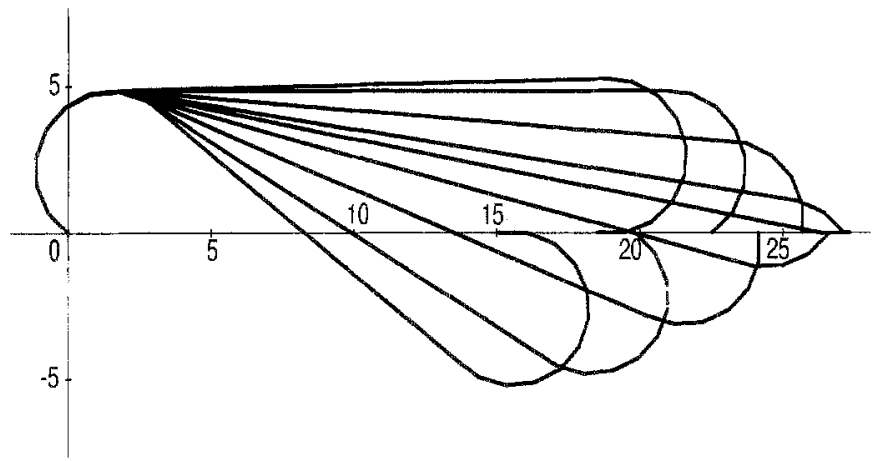

Fig. 12. Maximal reach curves with $\Psi_{0}=135^{\circ}, \Psi_{N}=-180^{\circ},-135^{\circ}, \ldots, 180^{\circ}$.

Figure 13 shows the same results as in Figure 12, but with all of the distances scaled so that $L=x_{N+1}=1$. This has the effect of changing $l$ from 1 to $1 / x_{N+1}$.

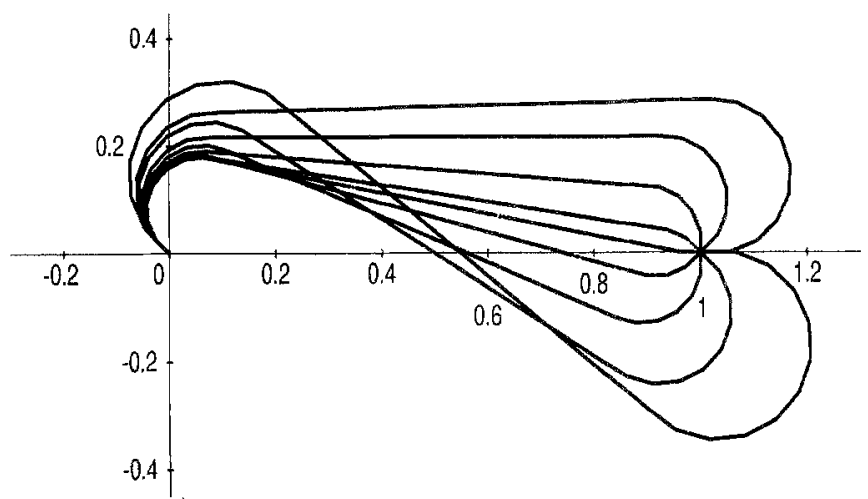

Fig. 13. Normalized maximal reach (minimal length) curves with $\Psi_{0}=135^{\circ}$, $\Psi_{N}=-180^{\circ},-135^{\circ}, \ldots, 180^{\circ}$.

Figure 14 shows the effect on varying $m$ while holding the other parameters constant at $\Psi_{0}=90^{\circ}, \Psi_{N}=-45^{\circ}, K=0.4, N=16, \alpha=1, \beta=1$. As $m$ increases, the curves converge to a circle-line-circle path. There is little change in the curves once $m$ is as large as 100 .

Figure 15 shows the same results as in Figure 14, but with all of the distances scaled so that $x_{N+1}=1$. Again, as $m$ increases, the curves converge to a circleline-circle path. 


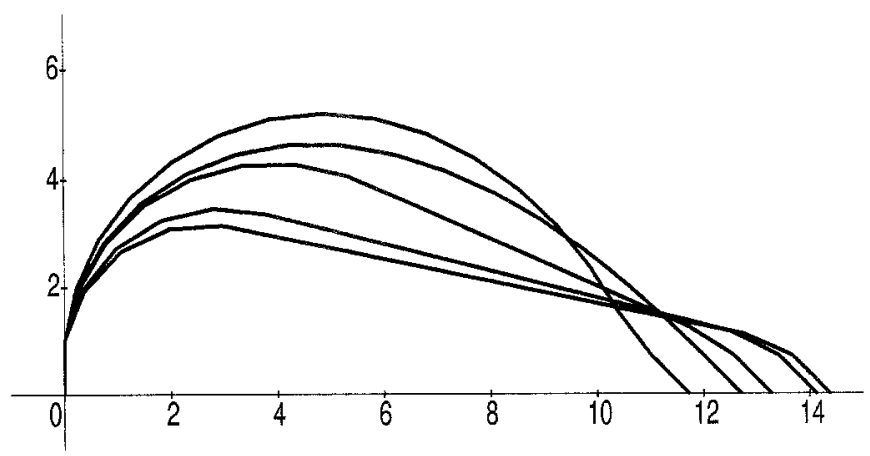

Fig. 14. Minimal length curves for turn-limited elastica with varying $m$. From top to bottom at $x=6$, these are the curves for $m=3,1,10,30$, and 100 respectively.

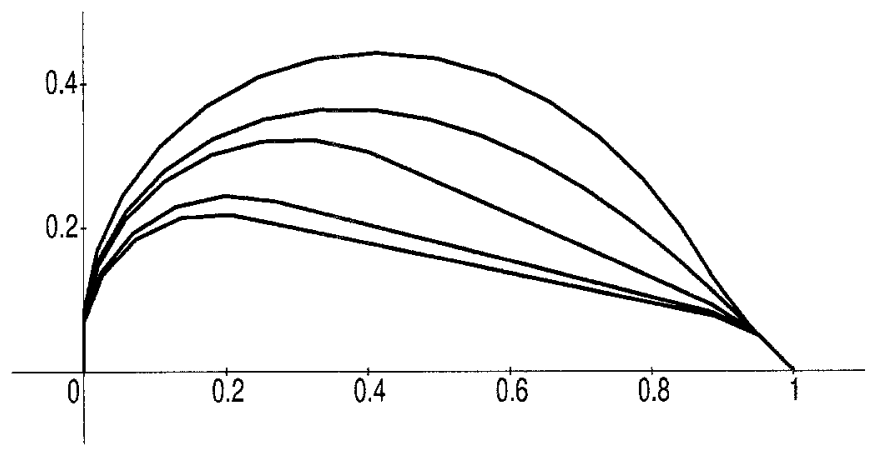

Fig. 15. Normalized similarity-invariant elastica with varying $m$. From top to bottom at $x=0.4$, these are the curves for $m=3,1,10,30$, and 100 , respectively.

\section{Conclusions}

We have introduced and discussed discrete versions of "minimal energy" and "minimum curvature variation" curve designs. We claim the discretizations provide excellent approximations to the continuous problems and may be very useful for a variety of CAD applications. Of course, other types of cost functions could and should be considered within the same framework.

The numerical work showed that the Newton-Raphson method was suitable for a wide variety of such problems, especially since good initial guesses could be provided, using the anticipated behavior of results stemming from theoretical studies of the continuous versions of the problems. A good initial guess was especially critical in the cases where the curvature penalty function approached a barrier function (not unexpectedly!). Extensions and comparisons to other numerical approaches are currently under investigation. 


\section{References}

1. L. Euler. Additamentum 'De Curvis Elasticis'. In Methodus Inveniendi Lineas Curvas Maximi Minimive Proprietate Gaudentes. Lausanne, 1744.

2. M. Born. Stabilität der elastischen Linie in Ebene und Raum. PhD thesis, Göttingen, Göttingen, Germany, 1906.

3. A. E. H. Love. The Mathematical Theory of Elasticity. Cambridge University Press, London, 1927.

4. M. Malcolm. On the computation of nonlinear spline functions. SIAM Journal of Numerical Analysis, 14:254-282, 1977.

5. I. D. Cooper. Curve interpolation with nonlinear spiral splines. IMA Journal of Numerical Analysis, 13:327-341, 1992.

6. S. Ullman. Filling in the gaps: The shape of subjective contours and a model for their generation. Biological Cybernetics, 25:1-6, 1976.

7. W. S. Rutkowski. Shape completion. Computer Graphics and Image Processing, 9:89-101, 1979.

8. B. K. P. Horn. The curve of least energy. ACM Transactions on Mathematical Software, 9:441-460, 1983.

9. M. Kallay. Plane curves of minimal energy. ACM Transactions on Mathematical Software, 12:219-222, 1986.

10. A. M. Bruckstein and A. N. Netravali. On minimal energy trajectories. Computer Vision, Graphics, and Image Processing, 49:283-296, 1990.

11. H. P. Moreton and C. H. Séquin. Scale-invariant minimum-cost curves: Fair and robust design implements. In Proceedings of Eurographics '93, pages C-473-C-484, Barcelona, September 1993.

12. D. Mumford. Elastica and computer vision. In C. L. Bajaj, editor, Algebraic Geometry and its Applications, pages 491-506. Springer-Verlag, New York, 1994.

13. E. H. Lee and G. E. Forsythe. Variational study of nonlinear spline curves. SIAM Review, 15:120-133, 1973 .

14. M. Golomb and J. Jerome. Equilibria of the curvature functional and manifolds of nonlinear interpolating spline curves. STAM Journal of Mathematical Analysis, $13: 421-458,1982$.

15. R. Bryant and P. Griffiths. Reduction for constrained variational problems and $\int \frac{\kappa^{2}}{2}$ ds. American Journal of Mathematics, 108:525-570, 1986.

16. K. Foltinek. The hamiltonian theory of elastica. American Journal of Mathematics, 116:1479-1488, 1994.

17. V. Jurdjevic. Non-euclidean elastica. American Journal of Mathematics, 117:93$124,1995$.

18. M. E. Mortensen. Geometric Modeling. John Wiley \& Sons, New York, 1985.

19. G. Farin. Curves and Surfaces for Computer Aided Geometric Design: A Practical Guide. Academic Press, Boston, 1988.

20. D. B. Parkinson and D. N. Moreton. Optimal biarc-curve fitting. Computer-Aided Design, 23:411-419, 1991.

21. C. L. Bajaj and G. Xu. NURBS approximation of surface/surface intersection curves. Advances in Computational Mathematics, 2:1-21, 1994.

22. L. E. Dubins. On curves of minimal length with a constraint on average curvature, and with prescribed initial and terminal positions and tangents. American Journal of Mathematics, 79:497-516, 1957.

23. B. W. Char, K. O. Geddes, G. H. Gonnet, M. B. Monagan, and S. M. Watt. Maple $\checkmark$ User's Guide. Watcom Publications Limited, Waterloo, Ontario, 1990. 